\title{
Importance of Cytometry for Clinical Diagnostics and Therapy
}

\author{
Ulrich Sack ${ }^{\mathrm{a}} \quad$ Jozsef Bocsi $^{\mathrm{b}} \quad$ Attila Tárnok ${ }^{\mathrm{b}}$ \\ a Department for Clinical Immunology and Transfusion Medicine, University of Leipzig, \\ b Pediatric Cardiology, Heart Center Leipzig, University of Leipzig, Germany
}

This issue of Transfusion Medicine AND Hemotherapy presents a small and by far not comprehensive overview of state of the art applications of cytometric analysis of clinical specimens in diagnosis and quality control being of special interest for transfusion specialists and hemotherapists. Focusing on applications in pediatrics, the seven papers of this overview address, among others, age-related normal values, neonatal infection, congenital heart disease and cord blood stem cells [1-7].

Flow cytometry is an analytical technology that allows quantifying cell parameters by measurement of optical characteristics. Analytical specificity is enhanced and improved by specific staining aided by fluorochrome-labeled specific molecules such as antibodies, enzyme substrates or dyes to measure cell physiology (e.g. membrane potential, intracellular $\mathrm{pH}$ or calcium). Analysis of cells by cytometric methods has been proved in the recent years as a highly standardizable and reliable method for biomedical research, process controlling in biotechnology, and quality assessment in transfusion medicine and organ transplantation and in immunological laboratory diagnostics [8].

Flow cytometry has several important features that make it extremely useful in quality control in hemotherapy and transfusion medicine, e.g. high-throughput (fast acquisition) and high-content measurement by determining polychromatic multiple parameters on the same cell (multiplexing [9], cytomics analysis [10]). Based on only minimal sample volume [5], extensive data are acquired that can be used to predict individualized disease courses (predictive medicine by cytomics). This concept indicates the hope that, by understanding cellular networks, cell systems can be identified that predict individual disease progression, outcome and response to therapy $[1,11,12]$. For example, in transplantation medicine cytometric analysis actually is used to explore reliable periph- eral biomarkers of relevant immune events that allows for monitoring of the immunosuppressive status of allograft recipients to predict pharmacodynamic drug effects and to individually balance immunosuppression [1].

By establishing more and more sophisticated technical approaches, such as multicolor analysis, slide-based cytometry and high-resolution detection of rare cell populations and weakly expressed molecules, a permanently growing number of applications for cytometric analysis has been introduced into routine diagnostic work $[1,6,8]$. Multicolor or polychromatic cytometric assays are advantageous as they allow for continuous monitoring of various parameters even in small children. Slide-based cytometry and related techniques offer unique tools to perform detailed immunophenotyping, enabling diagnostic procedures in very small sample volumes being two orders lower than those presently used in diagnostic routine [5]. Acquired data could have predictive value for individual risk assessment $[10,12]$.

By yielding quantitative data on the phenotypes and functions of leukocytes and lymphocytes, cytometry is an increasingly important tool in hemotherapy and transfusion medicine $[1,9$, 13]. Moreover, the concentrations of multiple soluble blood compounds can be determined cytometrically by multiplexed bead arrays [14]. Further indications of cytometry are the determination of physiologic and pathologic conditions (allograft recipients [1], infected neonates [3], children with protein losing enteropathy $[2,7])$, umbilical cord stem cell quality [4] and immune status of children [6].

In pediatrics where only minute blood volumes are at disposal the above mentioned advantages of flow cytometric analysis multiply each other. So it is not by coincidence that the main focus of the actual issue of Transfusion MEdicine AND HEMOTHERAPY is dedicated to the application of cytometric methods in children [2-7], summarizing some of the most in-

\begin{tabular}{ll}
\hline KARGER & @ 2007 S. Karger GmbH, Freiburg \\
Fax +49 7614520714 & Accessible online at: \\
$\begin{array}{l}\text { E-mail Information@Karger.de } \\
\text { www.karger.com }\end{array}$ & www.karger.com/tmh
\end{tabular}

Prof. Dr. Attila Tárnok

Pediatric Cardiology, Heart Center Leipzig, University of Leipzig

Struempellstraße 39, 04289 Leipzig, Germany

Tel. +49 341 865-2430, Fax -1143

E-mail tarnok@medizin.uni-leipzig.de 
teresting recent developments in the area of theoretical and applied clinical flow cytometry. Although these articles can reflect only a small part of cytometric methods, they give a general overview about diagnostically relevant applications. Fur- ther protocols are under investigation, and permanent improvement will allow to further reduce sample size, reagents' consumption as well as sample processing and acquisition time in the near future.

\section{References}

1 Barten JB, Gummert JF: Biomarkers in transplantation medicine: prediction of pharmacodynamic drug effects. Transfus Med Hemother 2007;34(3): 182-187.

2 Bocsi J, Lenz D, Sauer U, Wild L, Hess J, Schneider P, Bocsi J, Hambsch J, Schranz D Tárnok A: Inflammation and immune suppression following protein losing enteropathy after Fontan surgery detected by cytomics. Transfus Med Hemother 2007; 34(3):168-175.

3 Gille C, Orlikowsky T: Flow cytometric methods in the detection of neonatal infection. Transfus Med Hemother 2007;34(3):157-163.

4 Kamprad M, Kindler S, Schuetze N, Emmrich F: Flow cytometric immunophenotyping of umbilical cord and peripheral blood haematopoietic progenitor cells by different CD34 epitopes, CD133, P-glycoprotein expression and rhodamine-123 efflux. Transfus Med Hemother 2007;34(3):195-203. $\checkmark 5$ Laffers W, Schlenkhoff C, Pieper K, Mittag A, Tárnok A, Gerstner AOH: Concepts for absolute immunophenosubtyping by slide based cytometry. Transfus Med Hemother 2007:34(3):188-194.

6 Sack U, Fee, Tárnok A: Age related lymphocyte subset changes in the peripheral blood of healthy children - a meta-study. Transfus Med Hemother 2007;34(3):176-181.

7 Tárnok A, Bocsi J, Lenz D, Janousek J. Protein losing enteropathy (PLE) after Fontan surgery Clinical and diagnostical aspects. Transfus Med Hemother 2007;34(3):164-167.

8 Sack U, Tárnok A, Rothe G (Hrsg): Zelluläre Diagnostik. Basel, Karger, 2006.

9 Streit M, Ecker RC, Osterreicher K, Steiner GE, Bischof H, Bangert C, Kopp T, Rogojanu R: 3D parallel coordinate systems - a new data visualization method in the context of microscopy-based multicolor tissue cytometry. Cytometry A 2006;69A (7):601-611.
10 Tárnok A, Bocsi J, Brockhoff G: Cytomics - importance of multimodal analysis of cell function and proliferation in oncology. Cell Prolif 2006;39(6) 495-505.

11 Bocsi J, Mittag A, Sack U, Gerstner AO, Barten MJ, Tárnok A: Novel aspects of systems biology and clinical cytomics. Cytometry A 2006;69A(3): $105-8$.

12 Tárnok A, Brockhoff G: Cytomics emerging from cytometry. Cell Prolif 2006;39(5):335-338.

13 Shapiro HM, Perlmutter NG: Personal cytometers slow flow or no flow? Cytometry A 2006;69A(7): 620-630.

14 Sack U, Scheibe R, Wotzel M, Hammerschmidt S, Kuhn H, Emmrich F, Hoheisel G, Wirtz H, Gessner C: Multiplex analysis of cytokines in exhaled breath condensate. Cytometry A 2006;69A(3):169-172. 